\title{
Josef Koetschet - ein Schweizer in ärztlichen und diplomatischen Diensten der Türkei
}

\author{
Von Zdenko Levental
}

Nach den Napoleonischen Feldzügen im Orient machte sich im Ottomanischen Reich ein Anstieg der schon bestehenden Reformtendenzen bemerkbar ${ }^{1}$. Obwohl die feudalistisch-militaristische Gesellschaftsform dieser Weltmacht in ihren bestimmenden Strukturen vorwiegend noch bis nach dem Ende des Ersten Weltkriegs erhalten blieb ${ }^{2}$, machte die Pforte wiederholte Versuche, durch eine modernere Gesetzgebung und Umgliederung in Verwaltung und Armee, im Steuersystem, Schul- und Gesundheitswesen die ultrakonservativen Elemente innerhalb der eigenen herrschenden Klasse zu schwächen. Man hoffte, daß diese Reformen - teils radikale, öfters aber mehr kompromißartige Korrekturen - einem wichtigen Ziel dienen würden: den zentrifugalen Selbständigkeitsbewegungen in verschiedenen Ländern des großen Imperiums sowie dem wachsenden Befreiungswillen der nichtmoslemischen unterdrückten Völker entgegenzuwirken. Im Rahmen dieser Reformen und Versuche wurden auch die Aufstiegsmöglichkeiten in der Türkei für ausländische Fachleute immer günstiger. Im 19. Jahrhundert traf dieses Öffnen gegenüber Europa, nach dem Zusammenbruch der bürgerlichen Revolutionen von 1848 in einigen Staaten, mit einer Emigrationswelle verfolgter oder bedrohter europäischer Intellektueller zusammen. Unter diesen gab es auch Ärzte, besonders aus Polen und Ungarn, für welche die Türkei, vor allem ihre Armee, das einzige Ausweichsziel darstellte. Später, nach dem Berliner Kongreß 1878, mit dem weiter wachsenden expansionistischen Druck der Westmächte, wurden öfters auch offizielle Experten und Berater in die schon merkbar geschwächte Türkei entsandt.

Die Zahl der Schweizer Ärzte, die sich zu verschiedenen Zeitpunkten und aus verschiedenen Beweggründen in die Dienste der Türkei stellten, ist unbekannt, aber nach Schätzung von Kennern der Epoche nicht klein. Wenn in diesem Beitrag einer dieser Ärzte, Josef Koetschet, vorgestellt wird, so geschieht das vor allem aus dem Interesse des Autors für die Geschichte der schweizerischjugoslawischen medizinischen Beziehungen ${ }^{3}$, weil Koetschet den größten Teil seines Lebens und ärztlichen Wirkens in den südslawischen Ländern Bosnien und Herzegowina verbracht hat. Er wurde 1830 in Grellingen unweit von Delémont im Berner Jura geboren, wo es seit 1597 Daten über seine Vorfahren, die aus Holland stammen, gibt ${ }^{4,5,6}$. Unter den Koetschets gab es militante Anhänger der Französischen Revolution, aber auch verdienstvolle Geistliche. Josef, dessen 
Vater Statthalter in Laufen war, besuchte das Jesuiten-Gymnasium im elsässischen La Chapelle. Im Sommer $1848 \mathrm{nahm}$ er in Strasbourg an den revolutionären Geschehnissen teil. Im selben Jahre kam er als Mediziner nach Bern, wo er bald einer der Fahnenträger der «unruhigen» Studentenschaft wurde. Angeblich wegen Schwierigkeiten mit den Berner Behörden - zu einer Zeit, in der die Spannungen zwischen der Universität und der Regierung bedeutend waren ${ }^{7,8}$-, entschloß er sich, das Studium im Ausland fortzusetzen. Er ging nach Heidelberg, Wien und Paris, kehrte aber nach Bern zurück, um dort 1855 mit einer Dissertation aus dem Gebiet der orthopädischen Chirurgie zu doktorieren ${ }^{9}$.

Schon im selben Jahre verließ er wieder die Schweiz, um sich im militärischen Dienst der Türkei, die soeben in den Krimkrieg verwickelt war, zu verpflichten. Einige Zeit war er in Skutari (im heutigen Albanien). Nach einem Bericht traf ihn in Batum am Kaukasus ein anderer Schweizer Arzt: Alexander Schläfli. Während Koetschet der Schweizer Öffentlichkeit beinahe ganz unbekannt blieb außer Ebersolds Feuilleton ${ }^{4}$ scheint nie etwas über ihn in der Presse erschienen zu sein -, konnte sich Schläfli, obwohl er sehr jung starb, zu Lebzeiten einer breiten Publizität erfreuen, nicht nur dank seinen naturwissenschaftlichen Werken, sondern vor allem dank häufigen, ausführlichen Zeitungsberichten über seine Forschungsreisen ${ }^{10,11}$.

Entscheidend für Koetschets Zukunft war sein erfolgreicher Beginn als Leibarzt und Sekretär des ottomanischen Heerführers und Staatsmanns Omer Pascha Latas. Dieser schätzte ihn so hoch, daß er ihn neun Jahre in seinen Diensten behielt. Die Ausübung einer Doppelrolle, der ärztlichen und politischen, war für die weitere Laufbahn Koetschets bezeichnend. Mit Omer Pascha kam er 1861 in die von den Türken jahrhundertelang unterworfenen Balkan-Provinzen Bosnien und Herzegowina (mit einer slawischen Bevölkerung und seit 1918 zu Jugoslawien gehörend). Zu dieser Zeit wurde dort wieder einmal einer der häufigen Aufstände der beinahe rechtlosen und mißhandelten christlichen Leibeigenen mit Feuer und Säbel niedergeschlagen ${ }^{12}$. Zwei Jahre später wurde Koetschet zum ersten Stadt- und Polizeiarzt von Sarajevo ernannt, das nach Travnik ${ }^{13}$ der neue Sitz des Provinzgouverneurs war. Hier öffneten sich ihm neue Möglichkeiten, seine vielseitigen Begabungen zu entfalten.

Obwohl Koetschets Tätigkeit nur zum Teil seinem medizinischen Beruf galt, unternahm er auch in diesem Bereich einige wichtige Vorstöße. Es ist nicht gesichert, wie groß sein Anteil an der Eröffnung des ersten Militärspitals in der bosnischen Hauptstadt war; es ist aber sicher, daß er die Idee der Gründung des ersten Zivil-Krankenhauses gab und verwirklichte. Mit Geldern einer moslemischen religiösen Stiftung wurde der Bau 1866 beendet. Das Spital hatte nur 


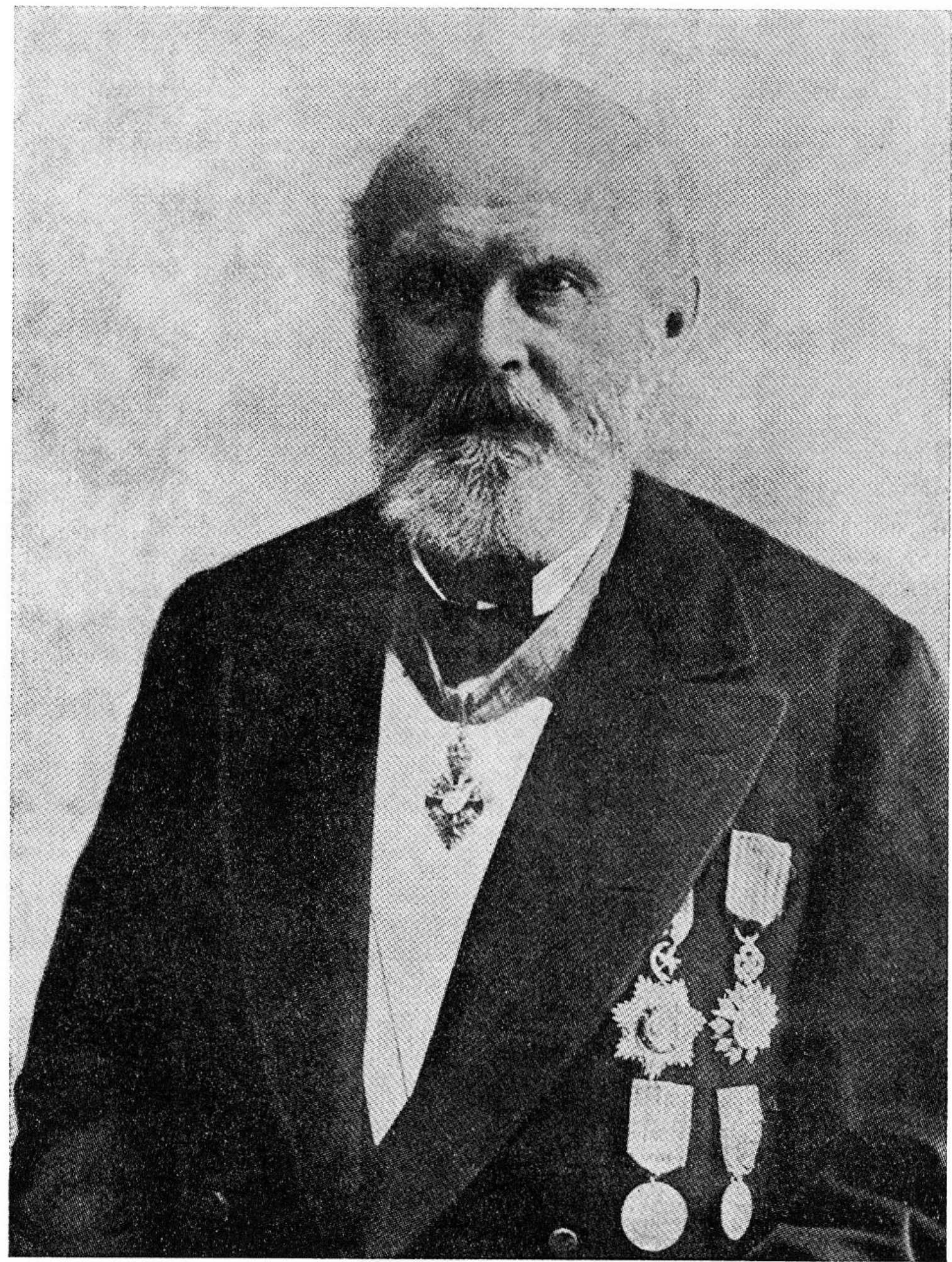

Josef Koetschet (1830-1898) 
32 Betten, aber auch eine kleine Apotheke, und konnte auch ambulatorische Patienten versorgen. Hauptsächlich diente es der ärmeren Bevölkerung sowie zur Behandlung von erkrankten Prostituierten ${ }^{14,15}$. Es hatte einen ständigen Arzt (zu Koetschets Zeit war dieser türkischer Herkunft), wobei Koetschet selbst vor allem eine Aufsichtsfunktion ausübte, was seinen überwiegend nicht-medizinischen Pflichten entsprach: er übte offiziell die Aufgaben eines Dragomans (Ủbersetzers) des Gouverneur-Wesirs aus, war jedoch weit über diese Tätigkeit hinaus vor allem politischer Sekretär und Berater des Provinzverwalters, oft mit Sondermissionen betraut und viel auf Reisen. Später, unter der österreichischen Besetzung Bosniens und der Herzegowina, wurde dieses Krankenhaus erweitert und 1894 in die erste psychiatrische Anstalt dieser Länder umgestaltet. Kurz nach der Besetzung Bosniens und der Herzegowina durch Österreich-Ungarn war Koetschet als Stadtphysikus einer der Anreger für den Bau des großen Landesspitals, das völlig westeuropäischen Maßstäben entsprach und 1894 seine ersten Kranken aufnehmen konnte ${ }^{16}$.

Koetschets Verdienste um die Verbesserung der Krankenversorgung in Sarajevo waren somit beachtenswert. Um so mehr muß man staunen, daß er in seinen ausführlichen politischen Memoiren ${ }^{17}$ kaum über sich als Arzt spricht, außer kurzen Erwähnungen einiger Maßnahmen gegen die Verbreitung einer CholeraEpidemie. Das gilt auch für seinen Biographen Georg Grassl ${ }^{18}$.

Das Staunen wird kleiner, das Verstehen größer, wenn man Koetschets beide Bücher liest, in denen ihr Autor als politischer Mensch par excellence erscheint. Es wird klar, daß diese Erinnerungen einem Mann gehörten, dessen Beruf zwar die Medizin war, aber dessen Neigungen und leidenschaftlicher Einsatz zum größten Teil der Tages- und der Staatspolitik galten: Einfluß auf die Taktik und die Entscheide der bosnisch-herzegowinischen Statthalter, diplomatische Missionen, ja sogar Versuche, von der Richtigkeit seiner Ideen und Voraussagungen die Schöpfer der osmanischen Innen- und Außenpolitik in Istanbul zu überzeugen.

Es gehört nicht zum Ziel und zum Rahmen dieses Beitrags, die Aufzeichnungen Koetschets und seine Rollen in den stürmischen Jahren des Verfalls der ottomanischen Herrschaft auf dem Balkan chronologisch und in Einzelheiten darzustellen und zu erläutern. Wir müssen uns darauf beschränken, aus seinen Memoiren einiges zu erwähnen, was für die Kenntnis und vielleicht auch das Verstehen seiner Persönlichkeit und seiner Orientierungen von Bedeutung und bezeichnend ist. Es muß in erster Linie gesagt werden, daß Koetschet selbst seine Position im politischen Leben nicht unterschätzte und von sich an einer Stelle als «Direktor für politische Angelegenheiten» spricht. In schwierigen Situationen und Krisen war er ein Berater seiner Arbeitgeber, der über zuverlässige Aus- 
künfte verfügte, die Lage scharfsinnig zu interpretieren verstand und einen weitreichenden Blick hatte. Durch jahrelange Loyalität hatte er sich Vertrauen verschafft, und sein Rat wurde in der Regel geschätzt. Trotzdem war sein Einfluß auf die zivilen und militärischen Machthaber Bosniens und der Herzegowina, wenn es um Grundsätzliches ging, sehr verschieden und oft nur bescheiden, da konservative Kräfte in den Ländern sowie die Intrigen und der sehr zögernde Reformwille in Istanbul liberaleren Vorschlägen der Walis (wie sich die Gouverneure auch nannten) entgegenarbeiteten. Dazu kam noch der sehr häufige Wechsel dieser hohen Beamten, die manchmal nur ein Jahr in Sarajevo blieben.

Koetschets nichtärztliche Tätigkeiten hatten ein vielfältiges Spektrum, von der Schlichtung örtlicher Konflikte bis zur Teilnahme an territorialen Abgrenzungskommissionen mit Serbien und Österreich. Er befand sich im Gefolge der Gouverneure bei Begrüßungen des österreichischen Kaisers Franz Joseph und führte als einzelner wiederholt vertrauliche und äußerst schwerwiegende Verhandlungen mit dem Fürsten Nikola von Montenegro. Dieser schätzte ihn noch von früheren Begegnungen und versuchte mehrmals, ihn für seinen eigenen Hof zu gewinnen. Es geschah auch, daß Koetschet vom Großwesir nach Istanbul zu persönlichen Berichten eingeladen wurde. Bei solchen Gelegenheiten - wie es auch in Sarajevo der Fall war - nahm er kein Blatt vor den Mund; doch wurde er zu seinem Bedauern in der Regel nur angehört, nicht auch erhört, da die Entscheide der Pforte meistens durch Einflüsse hinter den Kulissen erfolgten.

Als Vertrauensmann der Walis übernahm Koetschet auch so heikle Aufgaben, wie es die Hausdurchsuchung bei einem der Anführer der panslawischen Verschwörung in Mostar war, versuchte aber in solchen Situationen die Verdächtigten womöglich zu schützen. Wenn man hinzufügt, daß in den Jahren 1861-1878 während mehrerer Aufstände und Kriege der Südslawen gegen das Osmanische Reich ${ }^{19}$ Koetschets konsequente protürkische Haltung und sein Widerstand gegenüber den Befreiungsbewegungen allgemein bekannt war, ist es verständlich, daß die serbische und kroatische Presse ihn als Agenten der Unterdrücker brandmarkte. Er blieb trotzdem seiner Einstellung treu. Manche Stellen seiner Memoiren beweisen, daß es sich dabei nicht nur um ein rational-ideologisches, sondern auch um ein emotionelles Engagement handelte. So schreibt er z. B. oft «unsere Lage» oder «unser Volk»; er betont, daß ihn Osman Pascha «wie ein liebevoller Vater behandelt» habe, und denkt «mit wehmütigen Gefühlen» an einen anderen scheidenden Wesir. Es stimmt zwar, daß er sich mit harter Kritik gegen Fehler der lokalen Verwaltung und noch mehr der zentralen Regierung wandte, daß er veraltete Auffassungen, Bestechung und Gewalttätigkeiten verurteilte, doch setzte er sich grundsätzlich für die Wahrung des Status quo in den 
Balkan-Provinzen ein. Seine Identifikation mit der türkischen Sache war komplett und beständig. Und nur an einer Stelle in seinen Erinnerungen glaubte er etwas über eine mögliche Ambivalenz sagen zu müssen : «Ich erinnere mich heute noch des erblassenden Igumans ... und gestehe heute gern, daß ich mich als freiheitsliebender Christ eins fühlte mit ihm. ${ }^{20}$

Nach der österreichisch-ungarischen Okkupation 1878 hat sich Koetschet, zunächst als Stadtphysikus von Sarajevo und dann im Ruhestand, bis zu seinem Tode zwanzig Jahre später aus dem aktiven politischen Leben zurückgezogen. Zweimal seine Erinnerungen niederschreibend, zuerst französisch, dann nach der Vernichtung des Manuskripts durch einen Brand deutsch, hat er wohl im Geiste intensiv seine zahlreichen Begegnungen und Interventionen wiedererlebt, mit dem Bewußtsein, tief und endgültig mit dem unruhigen Bosnien verbunden zu sein. Er scheint nie daran gedacht zu haben, in die Schweiz zurückzukehren. Auch sein Sohn Theophil, ein hochbegabter Neuropsychiater, blieb der neuen Heimat treu ${ }^{21}$.

Der Versuch einer Analyse und Beurteilung von Koetschets Überzeugungen und Verhaltensweisen wäre nicht die Aufgabe des medizinischen, sondern des allgemeinen Historikers. Wir haben uns darauf beschränkt, die Umrisse eines biographischen Porträts zu zeichnen.

\section{Bibliographie und Anmerkungen}

1 Referate und Diskussionen des Seminars «Reformen im Osmanischen Reich im 19. Jahrhundert» unter der Leitung von Frau Prof. Dr. B. Mesmer und Dr. H. Fähndrich, Bern, November 1977 bis Februar 1978.

2 K. Steinhaus, Soziologie der türkischen Revolution, Frankfurt am Main 1969. Siehe besonders das Kapitel: Entfaltung von Innovationsprozessen im Osmanischen Reich, S. 26-45.

${ }^{3}$ Z. Levental, Beiträge zur Geschichte der schweizerisch-jugoslawischen medizinischen Beziehungen, Verh. des XIX. Intern. Kongr. für Geschichte der Medizin, Basel 1964, S. 452-460. - Derselbe: Médecins et infirmières de la Suisse - surtout de Genève - dans les guerres des peuples yougoslaves, Gesnerus 34 (1977), Heft 1/2, S.156-167. - Derselbe: Arnold Rikli aus Wangen (1823-1906) und seine «athmosphärische Kur», Jahrbuch des Oberaargaus 1977, S. 133-144.

4 F. Ebersold, Jos. Anton Koetschet. Ein Berner Arzt als Vertrauensmann der türkischen Regierung, Der Bund, No.405,406,407 vom 29.-31. August 1916.

5 P.-O. Bessire, Histoire du Jura Bernois et de l'ancien Evêché de Bâle, Porrentruy 1935.

6 Auszüge aus dem Taufregister der Pfarrei Pfeffingen (1734-1833) und aus den Registres de la Bourgeoisie de Delémont, die ich mit Dank von Staatsarchivar Dr. Hans Sutter aus dem Staatsarchiv des Kantons Baselland in Liestal und durch die freundliche Vermittlung von Herrn Rémy Koetschet, contremaître, Delémont, vom Secrétaire du Conseil Bourgeois, Delémont, Herrn Georges Chappuis, erhalten habe. 
7 R. Feller, Die Universität Bern 1834-1934, Bern 1935.

8 Fr. Haag, Die Sturm und Drang-Periode der Bernischen Hochschule 1834-1854, Bern 1914.

9 De la desarticulation médio-tarsienne. Thèse inaugurale présenté à la Faculté de Médecine de Berne par Joseph Koetschet Docteur en médecine. Berne, le 4 avril 1855.

10 Nachruf Schläflis von Albert Mousson in: Verhandlungen der Schweizerischen Naturforschenden Gesellschaft 1864, S. 326-386; Artikel in der Allg. Deutschen Biographie und im Hist.-biogr. Lexikon der Schweiz; umfassende Würdigung in: Burgdorfer Jahrbuch 1978, S. 9-99: Fritz Albrecht Leibundgut, Die Reisen des Dr. med. Alexander Schläfli von Burgdorf. Hierin über Koetschet: S.19f., 45 und Anmerkung 33. Schläfli nennt Koetschet einen ernsten wahrheitsgetreuen Mann.

11 A.M. (Albert Mousson), Dr. Alexander Schläfli und sein Reise-Unternehmen. SeparatAusgabe des Feuilleton der Neuen Zürcher Zeitung vom 20. und 21. Januar 1862. - Derselbe, Zweiter Bericht über das Reise-Unternehmen des Herrn Dr. Alexander Schläfli. Der naturforschenden Gesellschaft erstattet im April 1863. Auszug aus dem Feuilleton der Neuen Zürcher Zeitung, ohne Datum.

12 Fra Grga Martić, Zapamćenja (1829-1878), Zagreb 1906. Die Erinnerungen des Franziskaners Martić, der Politiker und Dichter war, sind eine wichtige Quelle für die Ereignisse dieser Zeit. An einigen Stellen spricht Martić auch über Koetschet und seine Rolle, so wie umgekehrt Koetschet auch Martić in seinen Memoiren oft erwähnt.

13 Über Travnik als Sitz der Gouverneure Bosniens und Schauplatz diplomatischer Spiele siehe auch den Roman Wesire und Konsuln des Nobelpreisträgers Ivo Andrić, Hanser, München [1961].

14 Über die Geschichte der Spitäler und des Gesundheitswesens überhaupt in Bosnien und der Herzegowina unter türkischer und österreich-ungarischer Herrschaft siehe vor allem: R. Jeremić, Prilozi istoriji zdravstvenih i medicinskih prilika Bosne i Hercegovine pod Turskom i Austro-Ugarskom, Beograd 1951. Diese Monographie enthält auch Daten über Koetschet und mehrere andere nichttürkische Ärzte in bosnisch-osmanischen Diensten. Siehe auch R. Zaplata, Zdravstvene prilike starog i današnjeg Sarajeva, Glas. jug. prof. društva XIX (1939), sv. 11 \& 12, sowie H. Curić, Zdravstvene prilike u BiH pred kraj turske vladavine, Zbornik I. kongresa za ist. zdrav. kult. Jugosl., Sarajevo 1970, S. 183 bis 186 (mit vielen bibliographischen Daten). Über ausländische Ärzte und die Entwicklung des Krankenhaus-Wesens siehe ferner: Z. Levental, im Kapitel «Povijest medicine», Medicinska enciklopedija, Zagreb 1963, Band 8, S. 224f.

15 Über die Einrichtungen des ersten Krankenhauses in Sarajevo und über Koetschets Initiativen: V. Kušan, Zdravstvene prilike starog Sarajeva, Beograd 1934. Einzelheiten über den Betrieb in diesem Krankenhaus bringt auch die autobiographische Schrift: G. Vučković, Robstvo u slobodi ili ogledalo pravde u Bosni, I-III, Novi Sad 1872.

16 Ein grundlegendes Werk zur Geschichte der Medizin Bosniens und der Herzegowina zu Ende des 19. Jahrhunderts ist: Das Sanitätswesen in Bosnien 1878-1901, Sarajevo 1903. Siehe auch das Jahrbuch des bosn.-herz. Landesspitals in Sarajevo für die Jahre 1894, 1895, 1896, Wien 1898.

17 J. Koetschet, Aus Bosniens letzter Türkenzeit, Zur Kunde der Balkanhalbinsel, Heft 2, Wien und Leipzig 1905. - Derselbe: Osman Pascha, der letzte große Wesier Bosniens, und seine Nachfolger, Zur Kunde der Balkanhalbinsel, Heft 9, Sarajevo 1909. 
18 G. Graßl, der Herausgeber von Koetschets Memoiren, in seiner Einleitung zu Aus Bosniens letzter Türkenzeit.

19 Von Schweizer Autoren, die über die sozialen und politischen Verhältnisse in Bosnien und in der Herzegowina zu dieser Zeit sowie die Aufstände und den ersten Krieg Serbiens und Montenegros gegen die Türkei geschrieben haben, siehe vor allem: W. Rüstow, Der Krieg in der Türkei. Zustände und Ereignisse auf der Balkanhalbinsel in den Jahren 1875 und 1876. Zürich 1877. - Über Friedrich Wilhelm Rüstow, einen deutschen Offizier und Flüchtling, der in der Schweiz eidgenössischer Oberst und hochgeschätzter Experte wurde, später auch erster Dozent für Kriegswissenschaften am eidgenössischen Polytechnikum in Zürich, schrieb P. Waldburger in: Allgemeine schweizerische Militärzeitschrift 121 (1955) 444-451.

20 J. Koetschet, Osman Pascha ..., S. 48.

21 Über Theophil Koetschet, der ein Schüler Kraepelins in München und dann Vizedirektor der Anstalt «Am Steinhof» bei Wien war, aber sich dann für eine leitende Stelle in Sarajevo entschloß: Zapisnik sastanka Sekcije za povijest medicine 1.i 23. 3. 1949, in Liječnički vijesnik, Zagreb 1949.

\section{Summary}

In the course of reform trends and modernization trials by the rulers of the Ottoman Empire during the 19th century, especially in its second half, among other foreign experts and professionals an increasing number of physicians has found open doors in Turkey and in other parts of the Empire. One of them was Josef Koetschet (1830-1898), a Swiss doctor, who joined the Turkish army services in 1855. He participated in the Crimean War and then accompanied Omer Pasha Latas to Bosnia and Herzegovina. These Balkan provinces were for centuries under Turkish occupation. From 1864 he was physician, interpreter, secretary and-more than all-political and diplomatic counselor of the provincial governors in Sarajevo, capital of Bosnia and Herzegovina.

Koetschet's two books of memoirs neglect quite completely his medical activities, although he has played a remarkable role in the foundation of the first hospitals in Sarajevo. His main interest was the internal and foreign policy of the provincial governors and even of the central government in Istanbul. Until Austria and Hungary occupied Bosnia and Herzegovina in 1878, Koetschet's political orientation was openly and completely proturkish. This provoked numerous attacks against him in the Croatian and Serbian press. After 1878 Koetschet has been appointed head physician of Sarajevo and retired from politics. He died in 1898. His son Theophil was an outstanding neuropsychiatrist trained in Germany and employed near Vienna, but he soon returned to Sarajevo feeling that Bosnia was his real homeland. His work as well as his father's life and publications are quite unknown in Swiss as well as in general medical history.

Prof. Dr. med. Zdenko Levental

Institut für Ausbildungs- und Examensforschung

Inselspital 14. c

3010 Bern 